\title{
La contrainte par le sol
}

\section{J. ROBERT}

Président de I'Union syndicale géotechnique

Directeur de EEG SIMECSOL
Tout projet de construction comporte des risques qui se sont amplifiés au fil des années. Ces risques, synonymes d'une maitrise souvent difficile du délai de réalisation et du coût final de l'ouvrage, résultent de l'évolution défavorable de plusieurs facteurs : délais d'études et de réalisation de plus en plus courts, ouvrage de plus en plus complexe mettant en jeu des méthodes de construction sophistiquées qui s'adaptent mal aux incertitudes et aléas, environnement de plus en plus sensible à toute perturbation. Ce constat justifie l'intervention d'un nombre croissant de spécialistes, d'oủ des problèmes d'interfaces plus nombreux et une coordination plus difficile.

Devant cette complexité croissante du projet. la géotechnique ne doit pas être oubliée car les exigences requises sur la qualité du sous-sol deviennent de plus en plus importantes en terme de fouille profonde, étanchéité, portance, déformations. Le sous-sol étant non visible, complexe et évolutif dans le temps, le bon géotechnicien est celui qui maitrise au mieux l'inconnu : pour cela, il accompagne le projet tout au long de sa conception et de sa réalisation par un enchainement de ses missions géotechniques conforme à la norme NF P 94-500. Ainsi, les risques résiduels seront assurables dans de meilleures conditions, par la mise en place de polices $\ll$ Dommage Ouvrage $n$ et $\approx$ Tous Risques chantier " basée, en particulier, sur l'intervention à bon escient du géotechnicien.

Mots-clés : missions (géotechniques), risques (géotechniques), zone d'influence géotechnique, maitrise des incertitudes du sol.

\section{Geotechnical missions progress}

NDLE: Les discussions Sur cet article sont acceptées jusqu'au $1^{\text {er }}$ octobre 2001.
Each civil work or building project contains risks which have been amplified, during the years. These risks, synonyms of difficulties for completing the construction at the initial cost and within the time limit, result of an unfavourable evolution of many factors: studies and execution time limit more and more short. constructions more and more complicated needing sophisticated methods with poor possibilities of adaptation in case of unforeseen geotechnical concitions, environment more and more sensitive to the disturbance. This statement justifles the intervention of an increasing number of specialists, with a great number of interface problems and a coordination more difticult. Considering the increasing complexity of the project, geotechnic shouldn't be forgotten because the requirements about ground behaviour become more and more important in term of deep excavation, tightness, bearing capacity, deformations. As the subsoif is invisible, complex and changing along the time, the good geotechnician controls at best the unknown factors: for that, he follows the project all, during its conception and its realisation by a geotecnical missions progress in conformity with the NFP 95-500 standard.

In this way, the residual risks are insurable with specific policies concerning construction damages (after completion) and all risks during works, according to the real geotechnical supervision.

Key words : (geotechnical) missions, (geotechnical) risks, geotechnical influence area. 
Aut fil des années, l'homme réalise des ouvrages de plus en plus complexes dans un environnement de plus en plus sensible à toute perturbation extérieure. La connaissance précise du contexte géotechnique et du comportement prévisionnel de l'ouvrage projeté (interaction sol-structure) tant en phase travaux qu'en phase d'exploitation devient ainsi primordiale pour assurer une bonne maîtrise des risques inhérents à tout projet de construction. Parmi ces risques, il doit être cité : - une part d'incertitude inévitable sur les caractêristiques du milieu naturel où s'inscrit l'ouvrage :

- le caractère aléatoire des actions subies :

- des incertitudes sur le comportement de l'ouvrage;

- l'hêtérogénéité des matériaux;

- le vieillissement mal connu de l'ouvrage.

Devant toutes ces incertitudes propres à la géotechnique et à l'ouvrage à construire, il est du devoir du spécialiste de sensibiliser le maittre d'ouvrage ou son représentant à une grande vigilance et dè l'inciter à se faire accompagner par un géotechnicien tout au long de la conception puis de la réalisation de l'ouvrage,

\section{1}

\section{Les spécificités de la géotechnique (complexe et évolutive)}

La géotechnique est la discipline qui étudie le comportement des terrains en relation ou non avec des ouvrages. Pour cela, elle s'appuie entre autres sur les différentes sciences de la terre suivantes : géologie, géophysique, hydrogéologie, mécanique des roches - mécanique des sols, géodynamique, rhẻologie des géomatériaux, géochimie.

Sa première spécificité est de se consacrer à l'étude du non-visible, donc d'un milieu naturel que l'on ne connait pas : ainsi, quelle que soit l'importance des investigations réalisées, la part du connu (par sondages, fouilles...) sera toujours faible par rapport au non-reconnu. Dans ces conditions, il est évident que les observations et relevés, réalisés lors de l'exécution des travaux de construction de l'ouvrage projetê, sont capitaux car bien plus nombreux et mieux répartis que les investigations faites dans le cadre des études : et pourtant, sur de très nombreuses opérations, ces observations et relevés ne sont pas faits, privant les constructeurs des données qui peuvent s'avérer fondamentales pour la réussite du projet.

Sa deuxième spécificité est d'étudier un milieu très complexe. Le sous-sol est constitué de terrains qui ne sont ni homogènes, ni isotropes, ni élastiques. Les couches des terrains sont souvent nombreuses et de disposition spatiale aléatoire, une même couche pouvant présenter des lithologies très différenciées. Souvent, le resserrement du maillage des sondages conduit à rendre plus complexe la structure du sous-sol, incitant parfois le donneur d'ordre à craindre l'intervention du géotechnicien qui conduit à rendre plus complexe le contexte géotechnique! Toute vérité géotechnique ne serait-elle pas bonne à dire? En fait, le sous-sol se prête mal à une modélisation qui est toujours simplificatrice et réductrice, alors que le problème qui survient est souvent dû à une hétérogénéité locale non décelẻe à temps !
En plus de la complexité au temps $t$ de la constitution du sous-sol, le facteur temps peut être source d'évolution irréversible ou non, parfois difficile à appréhender lors d'une étude forcément limitée dans le temps. Ainsi, parmi les principaux facteurs d'évolution dans le temps, il y a lieu de citer:

- les perturbations apportées par I'homme : exploitation de matériaux en carrières à ciel ouvert ou souterraines, réalisation d'ouvrages souterrains pouvant perturber les caractéristiques des terrains, pollution de certaines couches, remblaiement de fouilles qui deviennent ultérieurement non détectables :

- les phénomènes naturels liés à l'érosion et aux circulations d'eau, et mettant en jeu la stabilité de falaises ou de versants ;

- les secousses sismiques dont les effets sur certains terrains peuvent aller jusqu'à la perte totale de consistance: - la fluctuation des nappes contenues dans les terrains. qu'elle soit naturelle (influence de la pluviométrie) ou artificielle (pompages industriels, modification des circulations d'eau par création de barrages,...) :

- le gonflement ou le retrait de certains terrains argileux : les nombreux sinistres « sécheresse $»$ des années 80 sont là pour nous rappeler l'importance de ce paramètre directement lié à la pluviométrie ;

- la dissolution par l'eau de certains terrains (gypse, calcaire...), créant un réseau karstique évolutif ;

- les entrainements de fines sous l'effet d'écoulements naturels ou non.

Face à cette complexité naturelle, parfois amplifiée par le facteur temps, le géotechnicien a pour principales armes les investigations ponctuelles, d'où le danger des interpolations entre sondages face aux anomalies locales difficilement détectables tels le puits perdu. le talweg fossile, la carrière.

\section{Les spécificités de l'ouvrage à construire (plus vite, plus profond, moins cher)}

Les principales spécificités de l'ouvrage projeté qui influent sur sa conception et l'interaction sol-structure et qui sont donc à prendre en compte par le géotechnicien sont les suivantes

- son emprise au sol et les charges qu'il apporte : le volume de terrains mobilisés en dépend fortement; - la sensibilité de sa structure vis-à-vis des incertitudes géotechniques : incidences de la valeur des poussées s'exerçant sur l'infrastructure, influence des tassements et surtout des tassements différentiels :

- son implantation et sa profondeur dans le terrain, en ayant soin de bien définir le référentiel d'implantation et d'altimétrie :

- sa nature et ses sensibilités (points faibles) qui nécessiteront une adaptation des investigations et du suivi:

- ses phasages de construction qui imposeront des études d'interaction à chaque étape, même de courte durée. 


\section{Les méthodes de construction retenues (horlogerie)}

L'optimisation du choix technique des méthodes de construction suppose l'étude de l'influence des incertitudes géotechniques, principalement en termes de délais de réalisation et de coût final de l'ouvrage : il sera toujours conseillé de choisir les méthodes les moins sensibles à ces incertitudes pour éviter des situations de crise, La méthode sophistiquée qui serait mise en échec par une variation sensible de certains paramètres géotechniques est à proscrire si toute incertitude n'a pas pu être levée.

La méthode de construction elle-mème peut avoir une influence sur les caractéristiques géotechniques du site. Ainsi, les techniques d'amélioration de sols sont mises en ceuvre pour les améliorer et faciliter la conception de l'ouvrage, mais la méthode de construction peut elle-méme rendre le contexte géotechnique plus défavorable : par exemple remontée de la nappe phréatique par effet de barrage, passage aux caractéristiques résiduelles par apparition de grandes déformations...

Quelle que soit la méthode de construction retenue, les possibilités d'adaptations en cours de travaux doivent ètre étudiées, ainsi que leurs conditions de mise en ceuvre: observations à faire, moyens de contrôle, dispositions conservatoires..

En conclusion, la bonne maitrise des méthodes de construction mises en cuvre suppose une vérification en temps réel de la bonne concordance entre prévision et réalité du cornportement de l'ouvrage (auscultations), ainsi que de l'efficacité des procédés utilisés (par exemple pompages, soutènements, terrassements, améliorations de sol...).

\section{4}

\section{L'environnement (complexe, sensible, évolutif)}

L'impact sur l'environnement de tout projet est d'autant plus important à analyser tant au stade des travaux qu'au stade final de l'ouvrage construit que d'une part les ouvrages sont de plus en plus complexes, et d'autre part, l'environnement de plus en plus sensible.

Cet impact est lié en grande partie à l'extension de la zone d'influence géotechnique (ZIG), définie comme étant le volume de terrain au sein duquel il y a interaction entre d'une part le sol et les ouvrages environnants, et d'autre part, l'ouvrage du fait de sa réalisation et de son exploitation. Sa forme et son extension sont spécifiques à chaque site et à chaque ouvrage.

L'appréciation de la vulnérabilité des avoisinants, liée aux caractéristiques du sol situé en dehors de l'emprise du projet et à leur nature (structure et fondations) nécessite une étude spécifique.

La limitation de l'impact sur l'environnement passe en particulier par la maitrise des vibrations, des déplacements, des fluctuations de nappes... Tout doit être mis en cuvre pour réduire les incertitudes et assurer ainsi une meilleure maitrise de leurs conséquences potentielles sur l'environnement.

\section{5}

\section{Le rôle des divers intervenants (qui fait quoi)}

Le bon déroulement d'un projet, qui suppose une bonne maitrise des risques, nécessite des interfaces clairement définies entre les divers intervenants, toutes les fonctions nécessaires leur étant clairement attribuées. En particulier, vis-à-vis de la géotechnique, il y a lieu de s'assurer des points suivants :

- l'enchainement des missions géotechniques er accord avec l'évolution du projet (conception, travaux, maintenance)

- la définition des interfaces pour l'assurance de la qualitè

- l'intervention d'une maitrise d'cuuvre spécialisée pour les ouvrages spécifiques liés à la géotechnique et mettant en cuvre des techniques particulières :

- le suivi géotechnique lors des travaux, qui permet de s'assurer de la bonne adaptation des méthodes d'exécution retenues aux conditions gêotechniques réelles ou dans le cas de rencontre de conditions différentes de maitriser les conséquences sur le projet par la mise en cuvre à temps des adaptations nécessaires.

\section{6}

\section{La nature des contrats de construction (qui paie l'aléa)}

Le forfait pour les travaux liès à la géotechnique ne peut se concevoir que si le cadre géotechnique est bien connu : on ne peut forfaiter qu'une prestation bien définie.

Pour assurer une gestion optimale des risques, en limitant l'impact éconornique des incertitudes résiduelles, il est nécessaire

- de bien identifier les risques ;

- d'entreprendre les actions préventives pour les maîtriser :

- de définir les actions curatives pour en minimiser l'impact s'ils se réalisent ;

- de quantifier leur coût réaliste.

Ainsi, le risque (et donc son coût potentiel) pourra être attribué à la partie (maitre d'ouvrage, entrepreneur) qui est la plus influente sur la mise en œuvre réelle des actions préventives et curatives.

\section{7}

\section{L'enchaînement des missions géotechniques (un bon investissement)}

La nécessité de cet enchainement résulte des spécificités développées aux chapitres précédents. Il y a lieu d'insister sur les points suivants : 
- le reconnu en géotechnique est toujours modeste rapporté au volume de terrain concerné par le projet, il est bien moindre que celui utilisé pour les sondages d'opinion!:

- les risques liés à la géotechnique dépendent de nombreux facteurs: terrains, ouvrage, méthodes de construction, environnement, interfaces entre intervenants, contrats

- le risque zéro n'existe pas, mais pour une meilleure maîtrise de ses conséquences s'il se réalise, il faut:

- avant l'exécution des travaux

évaluer les incertitudes,

connaître la sensibilité de l'ouvrage et de l'environmement,

prévoir les dispositions constructives complémentaires à mettre en cuvre éventuellement.

\section{prévoir les adaptations possibles,}

signaler les risques inhérents (par exemple fissuration d'un bâti ancien lors de la construction en mitoyenneté d'un immeuble avec sous-sols) ;

- pendant l'exécution des travaux.

effectuer un suivi et un contrôle géotechnique en continu ;

- après les travaux :

mettre en ceuvre une maintenance adaptée au contexte géotechnique et à l'ouvrage.

Ainsi, s'il est utopique et économiquement irréaliste de lancer en début d'opération une étude géotechnique très poussée qu'on espérerait définitive, il est opportun de prévoir une mission du géotechnicien progressive et évolutive, comme celle du maître d'ceuvre qui se poursuit depuis l'étude de faisabilité jusqu'à l'achèvement de l'ouvrage. Seule une concertation sans cesse réactive entre le géotechnicien et les concepteurs et constructeurs permettra d'assurer la gestion optimale des risques du sol, à la satisfaction du maitre d'ouvrage.

Le géotechnicien, devant la complexité naturelle du sous-sol et les contraintes liées aux existants et avoisinants, procédera par ètapes successives, allant du général au particulier, de la reconnaissance globale à la reconnaissance locale, adaptant les investigations et les études à la sensibilité réelle du projet.

\section{8}

\section{Le contenu des missions géotechniques}

Par analogie avec les diverses missions de la maitrise d'cuvre accompagnant la réalisation d'une opération, le tableau I de la norme NF P 94-500 définit les différentes missions géotechniques types qui doivent être réalisées dans le cadre de la conception et de l'exécution d'un projet pour assurer une bonne maitrise des incertitudes et aléas géotechniques. Le schéma d'enchainement des missions géotechniques reporté en figure 1 de la norme établit la correspondance entre ces missions et les phases d'élaboration du projet par référence à la loi MOP (maîtrise d'cuvre publique).

Les missions géotechniques sont de deux natures différentes :

- les missions sans conseil, type G 0 : il s'agit de missions d'exécution de travaux d'investigations géotech- niques (sondages, essais, mesures) sur le terrain ou en laboratoire, selon un programme fourni (normalement dans le cadre d'une mission G 1 à G 5), et qui s'achève par la production d'un compte rendu factuel donnant les coupes des sondages, les procès-verbaux d'essais et les résultats des mesures en place ou en laboratoire. Ces missions excluent toute activité d'étude ou conseil :

- les missions avec conseil, type G1 à G 5 : il s'agit de missions géotechniques d'études et conseils, avec exploitation et interprétation des résultats des éventuelles missions $\mathrm{G} 0$ nécessaires à leur bon déroulement et réalisées par ailleurs (éventuellement par le mème géotechnicien).

Parmi les missions géotechniques avec conseil, on distingue:

- Les études de faisabilité géotechnique type G1, réalisées au stade des études préliminaires et de l'avantprojet (selon la loi MOP). Elles comprennent:

- l'étude préliminaire de faísabilité géotechnique G11 (stade des études préliminaires). Cette mission a pour objectif la connaissance générale du site sous l'angle géologique, hydrogéologique, géotechnique, et l'approche des principales contraintes qui en résultent pour une bonne adaptation d'un projet non encore défini au terrain. Limitée à la définition de certains principes généraux, la mission G 11 ne fournit pas les hypothèses géotechniques pour la justification du projet fil n'est pas encore connu précisément) ni des éléments de prédimensionnement;

- l'étude de faisabilité des ouvrages géotechniques G12 (stade des études d'avant-projet). Cette mission a pour objectif, après l'étude G11 et lorsque le projet est mieux défini :

en phase 1, de donner les hypothèses géotechniques du site à prendre en compte pour la justification du projet, quelques exemples types de fondations (encastrement et portance) et les principes généraux de construction des ouvrages géotechniques (notamment terrassements, soutènements, fondations, risques de déformation des terrains, dispositions générales vis-à-vis dés nappes et avoisinants). Cette ètude s'appuie sur les résultats d'une reconnaissance détaillée (mission $\mathrm{G} 0$ ) ;

en phase 2, de présenter des exemples dé prédimensionnement de quelques ouvrages géotechniques types envisagés (soutenements, rabattement de nappes, fondations, calcul de tassement, amélioration de sols), Cette étude sera reprise et bien entendu détaillée dans le cadre de l'étude de projet géotechnique G 2 .

Ces missions $\mathrm{G} 1$ excluent toute approche des quantités, des délais et des coûts d'exécution des ouvrages qui entre dans le cadre exclusif d'une mission d'étude de projet géotechnique G 2 .

- L'étude de projet géotechnique type G 2, réalisée au stade du projet et de "l'Assistance contrat travaux». Cette mission, intégrée ou non à celle de la maîtrise d'ceuvre, se décompose en deux phases :

- la phase 1 qui a pour objectif d'établir les notes techniques donnant les méthodes d'exécution retenues pour les ouvrages géotechniques (terrassements, soutènements, fondations, dispositions spécifiques vis-à-vis des nappes et des avoisinants), avec certaines notes de calcul de dimensionnement, une approche des quantités, délais et coûts d'exécution de ces ouvrages géotechniques ;

- la phase 2 qui a pour objectif d'établir les documents nécessaires à la consultation des entreprises pour l'exécution de ces ouvrages géotechniques (plans, notices techniques, cadre de bordereau des prix et esti- 


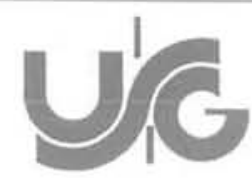

\section{CLASSIFICATION DES MISSIONS GÉOTECHNIQUES TYPES}

(Tableau 1 de la norme NF P 94-500 du 5 juin 2000)

L'enchaînement des missions géotechniques suit les phases d'élaboration du projet. Les missions G 1, G 2, G 3, G 4 doivent être réalisées successivement.

Une mission géotechnique ne peut contenir qu'une partie d'une mission type qu'après accord explicite entre le client et le géotechnicien.

\section{G 0 EXÉCUTION DE SONDAGES, ESSAIS ET MESURES GÉOTECHNIQUES}

- Exécuter les sondages, essais et mesures en place ou en laboratoire selon un programme défini dans des missions de type G 1 à G 5 ;

- Fournir un compte rendu factuel donnant la coupe des sondages, les procès verbaux d'essais et les résultats des mesures.

Cette mission d'exécution exclut toute activité d'étude ou conseil ainsi que toute forme d'interprétation.

\section{G 1 ÉTUDE DE FAISABILITÉ GÉOTECHNIQUE}

Ces missions $G$ I excltuent toute approche des quantités, délaís et coûts d'exécution des nuvrages qui entre dans le cadre excilusif d'une missinn d'étude de projet géotechnique $G 2$.

G 11 Étude préliminaire de faisabilité géotechnique

- Faire une enquête documentaire sur le cadre géotechnique du site et préciser l'existence d'avoisinants

- Définir si nécessaire une mission G 0 préliminaire, en assurer le suivi et l'expioitation des résultats :

- Fournir un rapport d'étude préliminaire de faisabilité géotechnique avec certains principes généraux d'adaptation de l'ouvrage au terrain. mais sans aucun élément de prédimensionnement.

Cette mission $G 11$ doit être suivie d'une mission G 12 pour définir les hypothèses géotechniques nécessuires à l'érablissement du projet.

G 12 Étude de faisabilité des ouvrages géotechniques (après une mission G 11)

Phase 1:- Définir une mission G 0 détnillée, en assurer le suivi et l'exploitation des résultats ;

- Fournir un rapport d'étude géotechnique donnant les hypothèses géotechniques à prendre en compte pour la justification du projet, et les principes généraux de construetion des ouvrages géotechniques (notamment terrassements, soutènements, fondations, risques de déformation des terrains, dispositions générales vis-à-vis des nappes et avoisinants).

Phase 2:- Présenter des exemples de pré dimensiornement de quelques ouvrages géotechniques types envisagés (notamment: soutènements. fondations, amélioration de sols).

Cette étude sera reprise et détaillée lors de l'étude de projer géonechnique (mission G 2),

\section{G 2 ÉTUDE DE PROJET GÉOTECHNIQUE}

Cette étude spécifique doit être prévue et intégrée dans le cadre de la mixssion de maûrrise d'rouvre.

Phase 1 :- Définir si nécessaire une mission $G 0$ spécifiquc, en assurer le suivi et l'exploitation des résultats :

- Fournir les notes techniques donnant les méthodes d'exécution retenues pour les ouvrages géotechniques (terrassements, soutènements. fondations, dispositions spécifiques vis-à-vis des nappes et avoisinants), avec certaines notes de calcul de dimensionnement, une approche des quantités, délnis et coûts d'exécution de ces ouvrages géotechniques.

Phase 2: - Établir des documents nécessaires à la consultation des entreprises pour l'exécution des ouvrages géotechniques (plans, notices techniques, cadre de bordereau des prix et d'estimatif, planning prévisionnel) ;

- Assister le client pour la sélection des entreprises et l'analyse technique des offres.

\section{ÉTUDE GÉOTECHNIQUE D'EXÉCUTION}

- Définir si nécessaire une mission $\mathrm{G} 0$ complémentaire, en assurer le suivi et l'exploitation des résultats :

- Étudier dans le détail les ouvrages géotechniques : notamment validation des hypothèses géotechniques, définition et dimensionnement (calculs justificatifs), méthodes et conditions d'exécution (phasages, suivi, contrôle).

Pour la mấtrise des incertitudes et aléas géolechniques en cours d'exécution, les misstons G 2 et G 3 doivent être sutvies d'une mission de suivi géotechnique d'exécution $G 4$.

\section{SUIVI GÉOTECHNIQUE D'EXÉCUTION}

- Suivre et adapter si nécessaire l'exécution des ouvrages géotechniques, avec définition d'un programme d'auscultation et des valeurs seuils correspondantes, antlyse et synthèse périodique des résultats des mesures :

- Définir si nécessaire une mission G 0 complémentaire, en assurer le suivi et l'exploitation đes résultats ;

- Participer à l'établissement du dossier de fin de travaux et des recommandations de maintenance des ouvrages géotechniques.

\section{G 5 DIAGNOSTIC GÉOTECHNIQUE}

\section{L'nbjet d'une mission $G 5$ est strictement limitatif. it né porte pas sur la totalité du projet ou de l'ouvruge.}

G 51 Avant, pendant ou après construction d'un ouvrage sans sinistre

- Définir si nécessaire une mission G 0 spécifique, en assurer le suivi et l'exploitation des résultats :

- Étudier de façon approfondie un élément géotechnique spécifique (par exemple soutènement, rabattement, etc.) sur la base des données géotechniques fournies par une mission G 12, G 2, G 3 ou G 4 et validées dans le cadre de ce diagnostic, mais sans aucune implication dans les autres domaines géotechniques de louvrage.

G 52 Sur un ouvrage avec sinistre

- Définir une mission G 0 spécifique, en assurer le suivi et l'exploitation des résultats :

- Rechercher les causes géotechniques du sinistre constate, donner une première approche des remèdes envisageables.

Une étude de projet géntechnique $G 2$ doit être réalisée ultérieurement. 


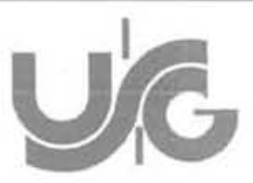

SCHÉMA D'ENCHAÎNEMENT DES MISSIONS GÉOTECHNIQUES

(Figure 1 de la norme NF P 94-500 du 5 juin 2000)

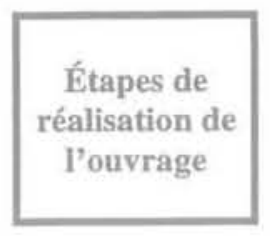

MISSIONS GÉOTECHNIQUES

\begin{tabular}{|c|c|c|c|c|c|c|}
\hline & & $\begin{array}{c}\text { Étude et suivi des } \\
\text { ouvrages } \\
\text { géotechniques }\end{array}$ & & $\begin{array}{c}\text { Exécution de } \\
\text { sondages, essais et } \\
\text { mesures } \\
\text { géotechniques }\end{array}$ & & $\begin{array}{l}\text { Diagnostic } \\
\text { géotechnique }\end{array}$ \\
\hline $\begin{array}{c}\text { Études } \\
\text { préliminaires }\end{array}$ & & $\begin{array}{l}\text { G } 11 \text { Étude } \\
\text { préliminaire de } \\
\text { faisabilité } \\
\text { géotechnique }\end{array}$ & \multirow{5}{*}{ G0 } & $\begin{array}{c}\text { G } 0 \text { préliminaire si } \\
\text { nécessaire (1) }\end{array}$ & \multirow{5}{*}{ G 5} & G 51 \\
\hline Avant-projet & G 1 & $\begin{array}{l}\text { G } 12 \text { Étude de } \\
\text { faisabilité } \\
\text { géotechnique } \\
\text { Phase } 1 \\
\text { Phase } 2\end{array}$ & & $\begin{array}{c}\text { G } 0 \text { détaillée } \\
\text { indispensable (1) }\end{array}$ & & G 51 \\
\hline $\begin{array}{l}\text { Projet } \\
\text { Assistance } \\
\text { Contrat } \\
\text { Travaux }\end{array}$ & G 2 & $\begin{array}{c}\text { Étude de projet } \\
\text { géotechnique } \\
\text { Phase } 1 \\
\text { Phase 2 }\end{array}$ & & $\begin{array}{l}\text { G } 0 \text { spécifique si } \\
\text { nécessaire (I) }\end{array}$ & & G 51 \\
\hline \multirow[t]{2}{*}{ Exécution } & G 3 & $\begin{array}{l}\text { Étude géotechnique } \\
\text { d'exécution }\end{array}$ & & \multirow[t]{2}{*}{ G 0 complémentaire } & & G 51 \\
\hline & G 4 & $\begin{array}{l}\text { Suivi géotechnique } \\
\text { d'exécution }\end{array}$ & & & & G. 52 \\
\hline
\end{tabular}
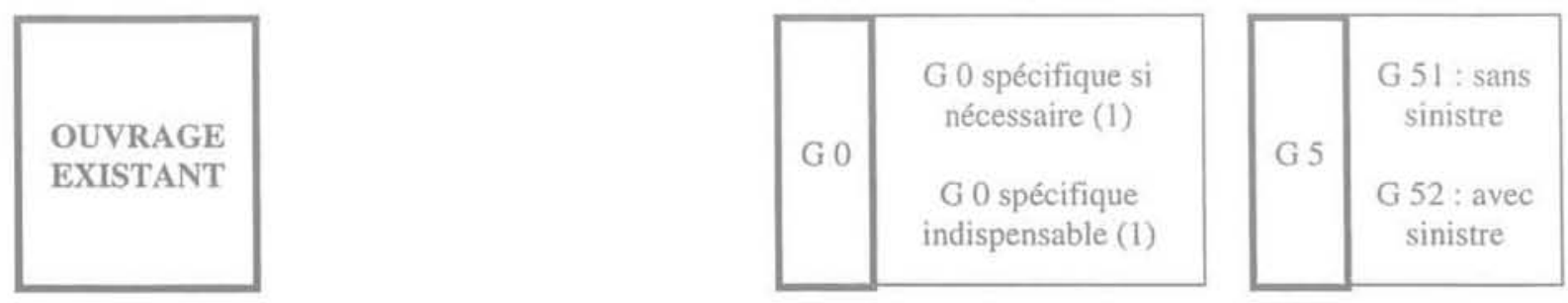

(1) À définir par le géotechnicien chargé de la mission. 
matif, planning prévisionnel) èt assister techniquement le client pour la sélection des entreprises spécialisées et l'analyse technique des offres.

La mission G 2 ne comprend pas les études détaillées de l'ouvrage géotechnique, qui feront l'objet de l'étude géotechnique d'exécution G 3 .

- L'étude géotechnique d'exécution type G 3, réalisée au stade de l'exécution de l'ouvrage. Cette mission, réalisée pour le compte du maître d'œuvre ou de l'entreprise selon le type de marché passé avec l'entreprise, a pour objectif d'étudier dans le détail les ouvrages géotechniques, en validant les hypothèses géotechniques, en définissant ces ouvrages et leur dimensionnement (calculs justificatifs), en établissant les méthodes et les conditions d'exécution (phasages, contrôles)

La mission G 3 ne comprend pas le suivi et contrôle d'exécution (mission G 4) nécessaire pour la maitrise des incertitudes et aléas géotechniques.

- Le suivi géotechnique d'exécution type G 4, réalisé au stade de l'exécution de l'ouvrage. Cette mission, indispensable en site géotechniquement complexe pour la maîtrise des aléas géotechniques en cours d'exécution. a de nombreux objectifs

- s'assurer que les travaux liés à la géotechnique sont réalisés conformément au projet ;

- maîtriser au mieux les incertitudes et aléas géotechniques subsistant après les études et qui sont susceptibles d'avoir des conséquences sur l'exécution et le comportement de l'ouvrage

- suivre pour adapter si nécessaire l'exécution des ouvrages liés à la géotechnique ;

- disposer au fur et à mesure de l'avancement des travaux de documents synthétiques précisant les conditions géotechniques rencontrées, le comportement des ouvrages en phase de construction et l'impact effectif sur les avoisinants.

C'est à ce stade que des économies importantes peuvent être réalisées grâce à une adaptation à temps des méthodes face aux aléas géotechniques rencontrés: comme en médecine, il est toujours plus aisé et efficace, de prévenir que de guérir.

- Le diagnostic géotechnique type G 5 qui ne porte pas sur la totalité de l'ouvrage. Il comporte deux missions différentes :

- avant, pendant ou après construction d'un ouvrage sans sinistre, cette mission G51 a pour objectif d'étudier l'incidence d'un élèment géotechnique spécifique, en complément des études géotechniques préalablement réalisées. Elle intervient dans le cadre de la mise au point d'une variante d'exécution pour optimisation ou adaptation d'un ouvrage, ou pour s'assurer du bon comportement futur d'un ouvrage face à des données géotechniques nouvelles;

- sur un ouvrage avec sinistre, cette mission G 52 a pour objectif d'en rechercher les causes géotechniques et de donner une première approche des remèdes envisageables, une étude de projet géotechnique devant être réalisée ultérieurement pour la mise au point du confortement de l'ouvrage.

\section{9}

\section{Conclusion}

L'enchaînement des missions géotechniques, en accompagnement de celles de la maîtrise d'œuvre, per- met une meilleure maîtrise des incertitudes et aléas du sol, donc des délais et des coûts d'exécution de l'ouvrage à construire. Ces missions géotechniques contribuent à une meilleure appréciation des obligations et des responsabilités du géotechnicien vis-à-vis des autres participants à l'acte de construire. Il ne sera jamais reproché à un géotechnicien de se heurter aux limites de la science mais il lui sera reproché de ne pas en informer ses cocontractants ou de laisser un flou sur le contenu de ses prestations exécutées.

Ainsi, la normalisation des missions devrait permettre de réduire la part de sinistralité des constructions trouvant son origine dans une mauvaise adaptation de l'ouvrage aux conditions géotechniques du site et résultant:

- soit d'impasses volontaires lorsque les conditions géotechniques sont connues avec une incertitude raisonnable:

- soit d'impasses involontaires lorsque la marge d'incertitudes sur les conditions géotechniques s'avère trop importante.

Pour inciter les maitres d'ouvrage à se faire accompagner dans leur propre intérêt, par un géotechnicien tout au long de la conception et de la réalisation de leur projet, des actions spécifiques au niveau des polices d'assurances sont souhaitables:

- pour la souscription d'une police « Dommage ouvrage $x$ :

- imposer une connaissance suffisante du contexte géotechnique,

- imposer une bonne adaptation du projet à ce contexte,

- imposer, dans les cas singuliers, un suivi et un entretien de l'ouvrage (maintenance) pendant son exploitation :

- pour la souscription d'une police « Tous Risques chantier 1$)$ :

- imposer le suivi géotechnique pendant les travaux.

En épilogue, en ce début de siècle, souhaitons que l'histoire ci-après d'un maître d'ouvrage satisfait devienne une histoire banale:

Un maitre d'ouvrage projette de construire un complexe touristique dans une large vallée des Alpes. Sur la base de son programme, il confie au géotechnicien une étude préliminaire de faisabilité géotechnique (G 11), afin de connaitre le contexte géotechnique général de site et pouvoir prendre en compte les contraintes en résultant pour une bonne adaptation du futur projet. gage d'une optimisation de son coût. Cette étude (G 11), qui s'est appuyée sur quelques sondages profonds $(G 0)$, conclut sur l'absence de certains risques majeurs (chutes de blocs, inondation...), et sur une qualité médiocre des terrains sur une profondeur dépassant $50 \mathrm{~m}$, avec risque de liquéfaction en cas de séisme, la nappe se situant vers $3,5 \mathrm{~m}$ de profondeur. Le géotechnicien conseille alors au maître d'ouvrage, d'une part, de prévoir des bâtiments de faible hauteur sur un niveau de sous-sol pour ne pas surcharger ces terrains médiocres et, d'autre part, de prévoir une amélioration des sols pour supprimer le risque de liquéfaction sous séisme et du même coup rendre envisageable une solution de fondations superficielles pour ce type de bâtiments.

Le maitre d'ouvrage demande à son architecte de concevoir un plan masse en conséquence, puis confie 
au géotechnicien l'étude de la faisabilité des ouvrages géotechniques correspondants. Cette étude (G 12), qui s'est appuyée sur des sondages géotechniques spécifiques $(\mathrm{G} 0)$ implantés au droit des bâtiments permet de conclure pour ces $\mathrm{R}+2$ sur sous-sol enterré à une solution optimale de fondation par radier général après amélioration de sols par injection solide pour maitriser le risque de liquéfaction et de tassements différentiels.

Après accord sur ces orientations, le maitre d'ouvrage confie au géotechnicien l'étude du projet géotechnique ( $G$ 2) qui permet, d'une part, de finaliser le projet d'amélioration des sols avec une approche des quantités, délais et coût correspondants et, d'autre part. d'établir les pièces de consultation des entreprises spécialisées puis d'assister le maître d'ouvrage pour retenir l'offre la mieux-disante.

L'entreprise spécialisée confie alors à un autre géotechnicien l'étude géotechnique d'exécution $(G 3)$ de cette amélioration de sols, avec notamment le dimensionnement détaillé, la définition des méthodes et des conditions d'exécution: phasages, suivi et contrôle interne de l'entreprise avec réalisation de sondages géotechniques ( $\mathrm{G} 0$ ).

Le géotechnicien du maître d'ouvrage assure le suivi géotechnique d'exécution (G 4), qui permet, par un programme de suivi et d'auscultation approprié, d'une part, de s'assurer de l'efficacité de l'amélioration de sols en maîtrisant au mieux les incertitudes et aléas géotechniques susceptibles d'avoir des conséquences sur le comportement futur des bâtiments, d'autre part, d'établir les recommandations de maintenance liées à ces ouvrages spéciaux (suivi altimétrique des bâtiments principalement).

Ainsi, le maitre d'ouvrage, en se faisant accompagner par un géotechnicien lors des diverses phases d'élaboration de son projet, a pu lancer l'exécution des superstructures dans les délais prévus, sans avoir connu de dépassement de budget pour ces travaux de fondations et d'infrastructure. Le succès de son opération a reposé largement sur cet enchaînement des missions, point fort de la norme NF 94-500 : il ne pouvait pas raisonnablement compter uniquement sur la chance comme le constructeur de la tour de Pise. 